\title{
Image Segmentation Using Signed Pressure Force Based Active Contour Model
}

\author{
${ }^{1}$ Sagar Chouksey, ${ }^{2}$ Mayur Ghadle, ${ }^{3}$ ShreyaSharma, ${ }^{4}$ Rohan Puranik \\ 1 Senior Consultant and Developer at Daffodil \\ Technologies Pvt .Ltd. \\ ${ }^{2}$ TIT Bhopal, ${ }^{3}$ OCT Bhopal, \\ ${ }^{4}$ Oriental College of Technology, Bhopa(M.P), \\ Electronics and Telecommunication Engineering. \\ sagar.infi@gmail.com,ghadlemayur23@gmail.com, \\ shreya.sharma.2491@gmail.com,rohan.pauranik.24@gmail.com
}

\begin{abstract}
A novel signed pressure force (SPF) based active contour model (ACM) is proposed in this work. It is implemented with help of Gaussian filtering regularized level set method, which first selectively penalizes the level set function to be binary, and then uses a Gaussian smoothing kernel to regularize it. The advantages of this method are as follows. First, a new region-based signed pressure force (SPF) function is proposed, which can efficiently stop the contours at weak or blurred edges. Second, the exterior and interior boundaries can be automatically detected with the initial contour being anywhere in the image. Third, the proposed SPF with ACM has the property of selective local or global segmentation. It can segment not only the desired object but also the other objects. Fourth, the level set function can be easily initialized with a binary function, which is more efficient. The computational cost for traditional re-initialization can also be reduced.
\end{abstract}

Index Terms-Active Contour Model (ACM); Geometric Active Contour (GAC); Signed Pressure Force (SPF) Function; Energy Functional etc.

\section{Council for Innovative Research}

Peer Review Research Publishing System

\author{
Journal: INTERNATIONAL JOURNAL OF COMPUTERS \& TECHNOLOGY \\ Vol 12, No.1
}

editor@cirworld.com

www.cirworld.com, member.cirworld.com 


\section{Introduction:}

Many standard methods of image segmentation have been introduced, where we stressed the importance and paramount role of regimentation in almost all aspects of image analysis. Unsurprisingly, segmentation of image methods are rapidly developing as novel and more powerful approaches continue to appear. New approaches must be able to cope with everincreasing numbers of image datasets, of increasing size of individual image, as well as with increasing dimensionality.

The methods presented have several other features in common they were introduced relatively recently, each called for a paradigm shift when introduced, and all of them proved valuable, flexible, and highly applicable. A variety of segmentation techniques reported in the literature, we will explain here a number of selected techniques using mean shift segmentation, fuzzy connectivity, deformable models, gradient vector flow, graph search, graph cuts. These methods bring a number of new concepts, clearly demonstrating the departure of newly-introduced segmentation methods [1].

\section{Literature Survey}

A number of research papers have been studied related to medical image processing. Research in medical informatics concentrates primarily on integrating computer technology to improve all areas of the field from education to diagnosis and treatment. Extensive study has been made and many techniques have been proposed among which the active contour model (ACM) [2] is one of the most successful methods. The basic idea in active contour models (or snakes) is to evolve a curve, subject to constraints from a given image, in order to detect objects in that image. They are classified as either parametric active contours or geometric active contours according to their representation and implementation. Geometric active contours have many advantages over parametric active contours. Parametric active contours have been reproduced in geometric active contours, the relationship between the two has not always been clear.

Active contours, also called snakes, are among the most important tools in computer vision. They were introduced in [3] and have been widely used for image analysis tasks such as object boundary detection. Despite its success, the original parametric active contour model has some noticeable drawbacks. First, it depends not only on the intrinsic properties of the contour but also on its parameterization; thus, it is a non-geometric model. Second, it cannot naturally handle changes in the topology of the evolving contour; significant progress towards topologically adaptable parametric snakes has been done recently. These drawbacks of standard active contours were addressed by geometric active contours, introduced in [4]. An important development has been the introduction of geodesic active contours [5]. Augmenting the edge-based geodesic active contours with other region-based visual cues has led to many powerful geometric active contour models. Many region-based geometric active contours, have been inspired by the piecewise smooth Mumford-Shah image model [6].Another class of region-based geometric active contour models builds on the statistical region competition and important developments include geodesic active regions [5]. Most implementations of geometric active contour models build on the level-set method of Osher and Sethian [7], in which the active contour is given implicitly as the zero level set of a scalar embedding function defined on the whole image domain. Level sets in implementing certain geometric active contour models, but they are not as generally applicable as level sets. Despite the advantages of the level-set method, its computational cost can be high, rendering its utilization for time-critical applications problematic. To restrict computations to the neighborhood of the evolving contour, narrowband methods in conjuction with reinitialization techniques from the level-set technology [8] have been used.

In [9] they propose an efficient multigrid algorithm for level-set implementations of geometric active contour models. First, they demonstrate the utility of multigrid curve evolution algorithms in efficiently solving a variety of additional computer vision models which encompass geometric active contours ideas, such as the Mumford-Shah and the region competition/geodesic active regions models; this is achieved in the framework of implicit-explicit schemes. Second, we elaborate on the optimal design of the multigrid solver components, depending on the numerical properties of the specific problem one solves. In [10] authors develop a precise relationship between the two which includes spatially-varying coefficients, both tension and rigidity, and non-conservative external forces. This relationship highlights the Lagrangian nature of parametric active contours and the Eulerian nature of geometric active contours. The result is a very general geometric active contour formulation for which the intuitive design principles of parametric active contours can be applied.

In [11] the authors presented a scheme for the detection of object boundaries. The proposed approach is based on the relation between active contours and the computation of geodesics or minimal distance curves. This geodesic approach for object segmentation allow us connecting classical "snakes" based on energy minimization and geometric active contours based on the theory of curve evolution. Previous models of geometric active contours are improved, allowing stable boundary detection when their gradients suffer from large variations, including gaps. In traditional snake algorithm there are some problems associated with initialization and poor convergence to boundary concavities, which limits their utility. There are two key difficulties with parametric active contour algorithms. First, the initial contour must, in general, be close to the true boundary or else it will likely converge to the wrong result. The second problem is that active contours have difficulties progressing into boundary concavities.In [12], authors proposed a new external force for active contours, largely solving both problems. This external force, which we call gradient vector flow (GVF), is computed as a diffusion of the gradient vectors of a gray-level or binary edge map derived from the image. GVF has a large capture range and is able to move snakes into boundary concavities. The GVF and Geodesic Active contour approaches eliminate the problems with uneven control points and self-crossing and can easily be extended to higher dimensional applications. However, they still have the problem of local minima.

In [13], authors presented a graph cuts based active contours (GCBAC) approach to object segmentation problems Consequently, it has the following advantages. (1). It has the ability to jump over local minima and provide a more global result. (2). Graph cuts guarantee continuity and lead to smooth contours free of self-crossing and uneven spacing problems. Therefore, the internal force which is commonly used in traditional energy functions to control the smoothness is no longer needed, and hence the number of parameters is greatly reduced. (3). this approach easily extends to the segmentation of three and higher dimensional objects. In addition, the algorithm is suitable for interactive correction and is shown to 
always converge.As given in [14] the basic idea in active contour models (or snakes) is to evolve a curve, subject to constraints from a given image, in order to detect objects in that image. Ideally, we begin with a curve around the object to be detected, and the curve then moves normal to itself and stops at the boundary of the object. In traditional level set methods, the level set function $\varphi$ can develop shocks, very sharp and/or flat shape during the evolution, which makes further computation highly inaccurate. To avoid these problems, a common numerical scheme is to initialize the function $\varphi$ as a signed distance function before the evolution, and then "reshape" (or "re-initialize") the function $\varphi$ to be a signed distance function periodically during the evolution. Reinitialization has been extensively used as a numerical remedy in traditional level set methods.

In [15], a new model is proposed for active contours to detect objects in a given image, based on techniques of curve evolution, Mumford-Shah functional for segmentation and level sets. This model can detect objects whose boundaries are not necessarily defined by gradient. In the level set formulation, the problem becomes a "mean-curvature flow"-like evolving the active contour, which will stop on the desired boundary. However, the stopping term does not depend on the gradient of the image, as in the classical active contour models, but is instead related to a particular segmentation of the image. In this authors provided a numerical algorithm using finite differences. Finally, authors presented various experimental results and in particular some examples for which the classical snakes methods based on the gradient are not applicable. Also, the initial curve can be anywhere in the image, and interior contours are automatically detected.

\section{Problem Statement}

The only drawback of this model discussed above is the existence of local minima in the active contour energy, which makes the initial guess critical to get satisfactory results. We will prove results to determine the existence of a global minimum of the SPF based active contour model. From a numerical point of view, we propose a new practical way to solve the active contour propagation problem toward object boundaries through formulation of the minimization problem. A new region-based signed pressure force (SPF) function is proposed, which can efficiently stop the contours at weak or blurred edges. Second, the exterior and interior boundaries can be automatically detected with the initial contour being anywhere in the image. Third, the proposed SPF based ACM has the property of selective local or global segmentation. It can segment not only the desired object but also the other objects. Fourth, the level set function can be easily initialized with a binary function, which is more efficient.

\section{Flow Chart of Proposed Method:}

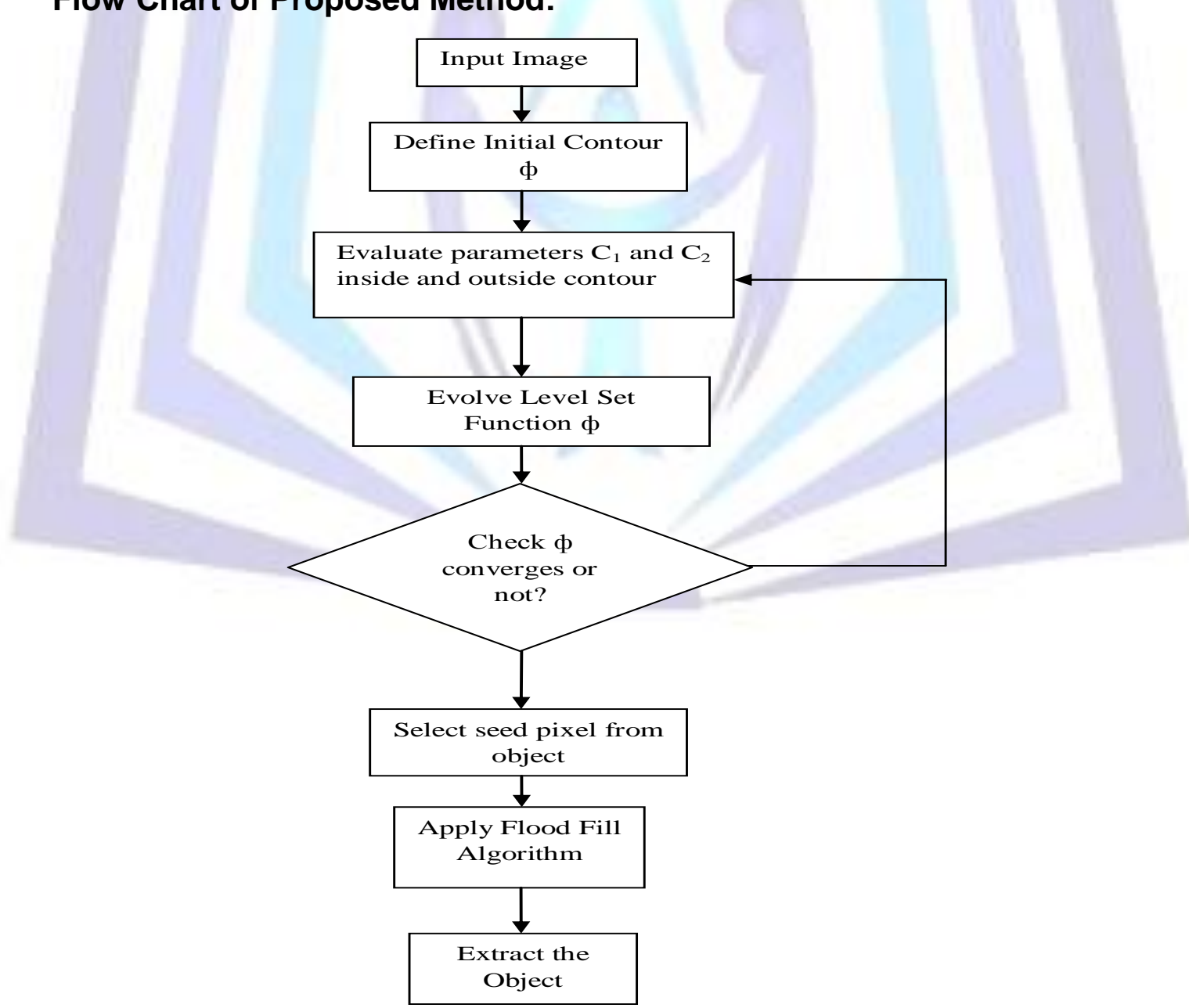




\section{Object Segmentation Algorithm Using SPF}

1. Select input image from database.

2. Define initial level set function $\phi$ to be binary function as:

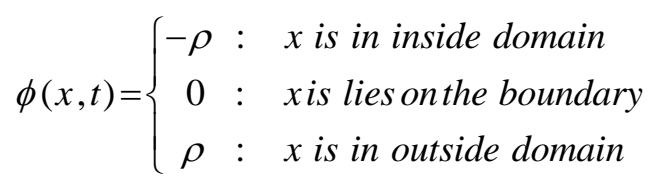

Compute parameter as: $\mathrm{c}_{1}(\phi)$ and $\mathrm{c}_{2}(\phi)$

3. Expansion or shrinkage of level set function $\phi$ according to energy minimization or maximization.

4. Using Gaussian filter regularize the level set function.

5. If level set function converges then stop otherwise go to step 3.

6. If it converges select a seed pixel within object contour.

7. Apply flood fills algorithm for object segmentation from background.

\section{Experimental Results}

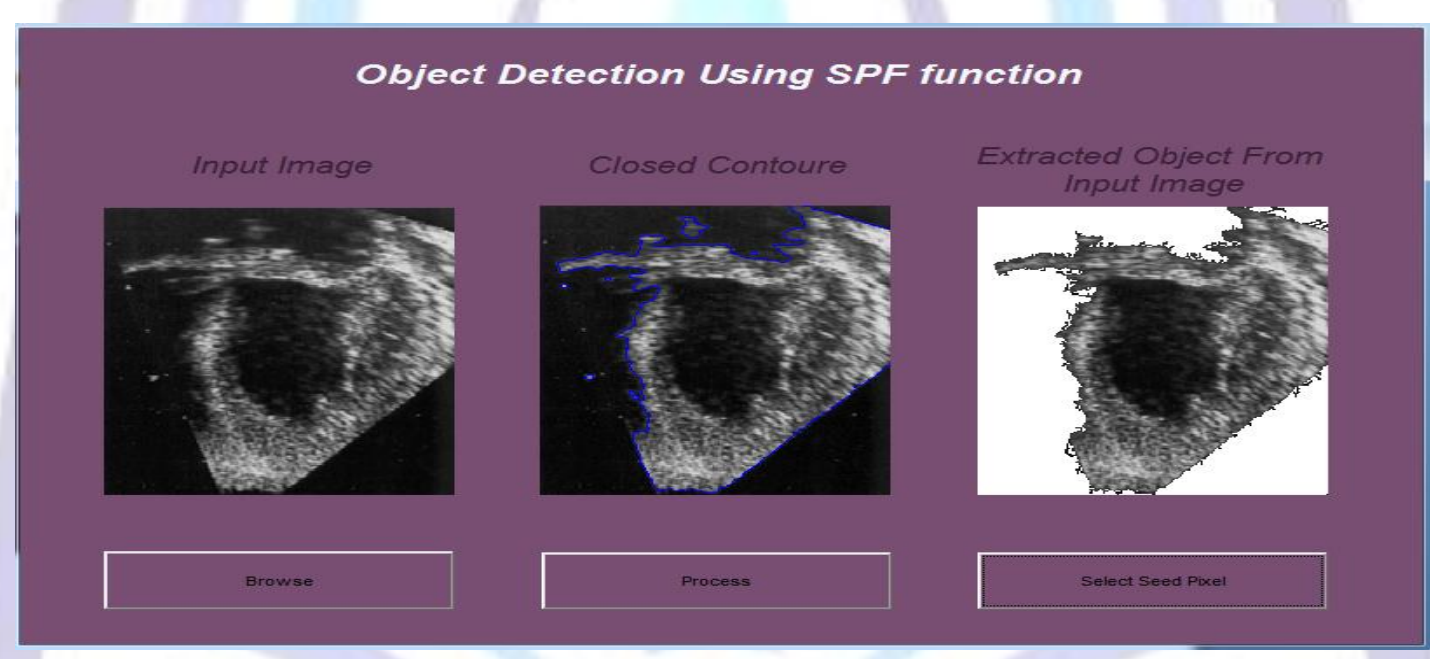

Figure 2: Demonstrates the global segmentation property of SPF based ACM method. (a) Input image (liver) (b) Object contours (c) Extracted object.

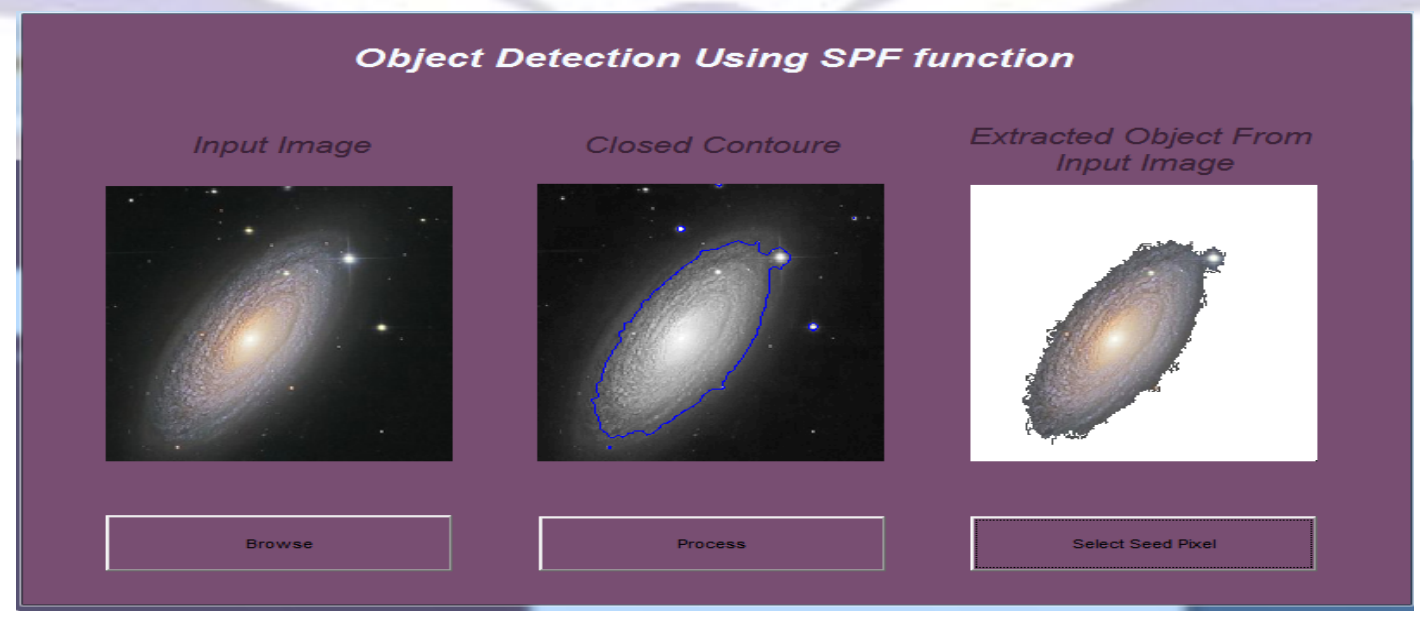

Figure 3: Demonstrates the global egmentation property of SPF based ACM method. (a) Input image (galaxy) (b) Object contours (c) Extracted object flood fill. 


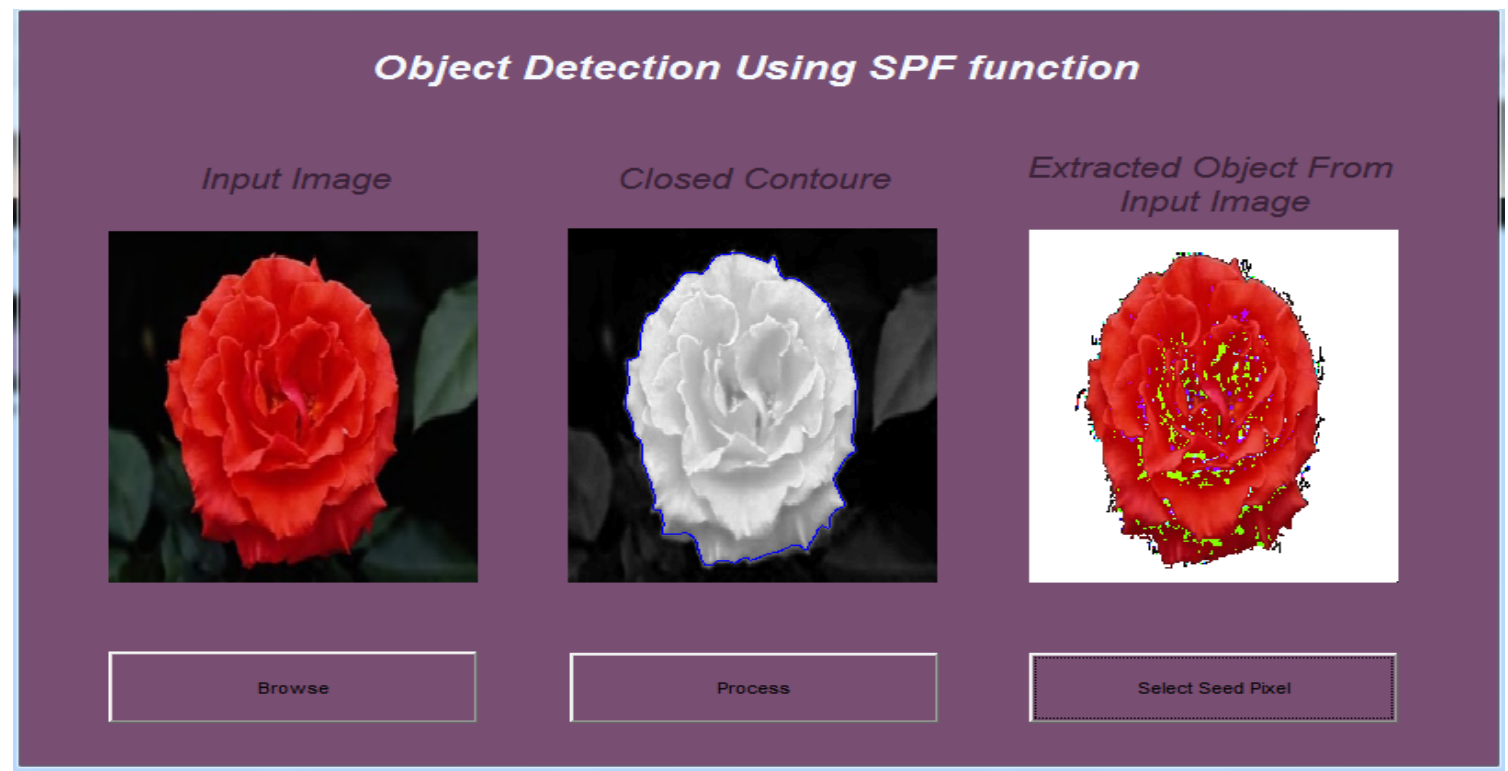

Figure 4: Demonstrates the global segmentation property of SPF based ACM method. (a) Input image (galaxy) (b) Object contours (c) Extracted object using flood fill.

\section{Conclusion:}

SPF based active contour model is a well-known image segmentation model which is used in various medical image processing. Its success is based on strong theoretical properties and efficient numerical schemes. This segmentation model is the existence of local minima in its functional energy, which makes critical the initial contour to extract meaningful objects lying in images. This work a new approach to determine a local/global minimum of the SPF based active contour model in order to become independent of the initial position of the contour. We think that this new approach can have numerous applications in the image processing tasks previously mentioned. This model is based on the existence of global minimizers to our segmentation variational models. We determined not one but several global minima of the active contour model, which looks to be a drawback. However, all global solutions are close to each other because the minimizations of gradients are very close to binary functions and hopefully it will give better results in comparison of other existing methods.

\section{References:}

[1]. $\quad$ R.C. Gonzalez and R.E. Woods, "Digital Image Processing," Addison Wesley, Reading MA, 1992.

[2]. N. Xu, N. Ahuia, R. Bansal, "Object segmentation using graph cuts based active contours," Computer Vision and Image Understanding, vol. 107, pp. 210-224, 2007.

[3]. M. Kass, A. Witkin, and D. Terzopoulos, "Snakes: Active contour models," Int. J. Comput. Vis., vol. 1, no. 4, pp. 321-331, 1988.

[4]. $\quad$ R. Malladi, J. A. Sethian, and B. C. Vemuri, "Shape modeling with front propagation: a level set approach," IEEE Trans. Pattern Analysis Machine Intelligence, vol. 17, no. 2, pp. 158-175, Feb. 1995.

[5]. V. Caselles, R. Kimmel, and G. Sapiro, “Geodesic active contours,” Int. J. Comput. Vis., vol. 22, no. 1, pp. 61-79, 1997.

[6]. D. Mumford and J. Shah, "Optimal approximations by piecewise smooth functions and associated variational problems," Commun. Pure Appl. Math., vol. 42, no. 5, pp. 577-685, 1989.

[7]. S. Osher and J. A. Sethian, "Fronts propagating with curvature dependent speed: Algorithms based on HamiltonJacobi formulations," J. Comput. Phys, vol. 79, pp. 12-49, 1988.

[8]. J. A. Sethian, "Level Set Methods and Fast Marching Methods," 2nd ed. Cambridge, U.K.: Cambridge Univ. Press, 1999.

[9]. P. George, P. Maragos, "Multigrid Geometric Active Contour Models," IEEE Transactions on Image Processing, vol. 16, no. 1, pp. 229-241, January 2007.

[10]. C. Davatzikos, J.L. Prince, "An active contour model for mapping the cortex," IEEE Transaction on Medical Imaging, vol. 14, pp. 65-80, 1995.

[11]. V. Caselles, R. Kimmel, G. Sapiro, "Geodesic active contours," International Journal of Computer Vision, vol. 22, no. 1 , pp. 61-79, 1997.

[12]. C. Xu, J.L. Prince, "Snakes, Shapes, and gradient vector flow," IEEE Transaction on Image Processing, vol. 7, pp. 359-369, 1998. 
[13]. N. Xu, N. Ahuia, R. Bansal, "Object segmentation using graph cuts based active contours," Computer Vision and Image Understanding, vol. 107, pp. 210-224, 2007.

[14]. V. Caselles, R. Kimmel, G. Sapiro, "Geodesic active contours," International Journal of Computer Vision, vol. 22, no. 1 , pp. 61-79, 1997.

[15]. D. Mumford, J. Shah, "Optimal approximations of piecewise smooth functions and associated variational problems," Communication of Pure Appl. Math. vol. 42, pp. 577-685, 1989.

\section{Author Biography:}

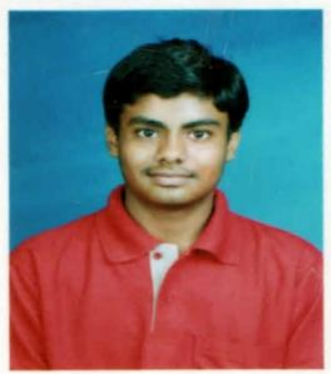

Sagar Chouksey received BE degree in

Electronics and Telecommunication Engineering

from

Lakshmi Narain College of Technology, Bhopal (M.P) in 2011.

He then joined Infosys Technologies Ltd

and held the position of System Engineer

from 2011-2013.

Now, he is working as a Senior Consultant and

Developer at Daffodil

Technologies Pvt .Ltd. 